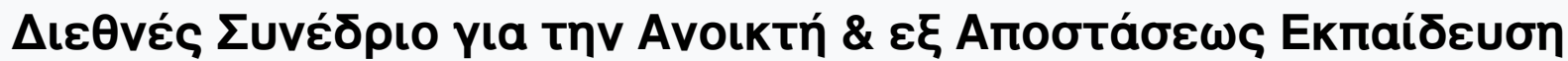

Tóp. 6, Ap. 2B (2011)

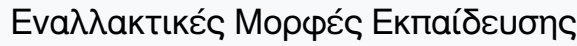

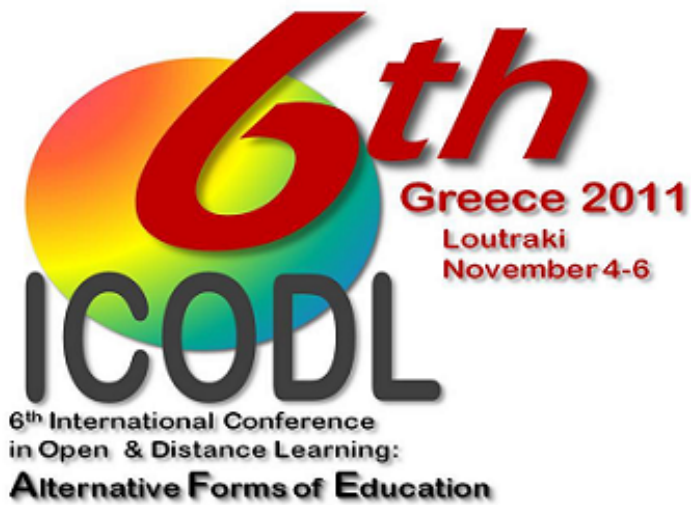

TOMOE B

PART / MEPOE B

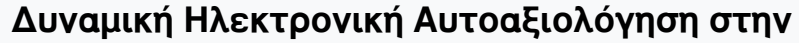
A.E६.A.E.

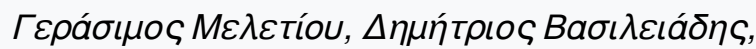

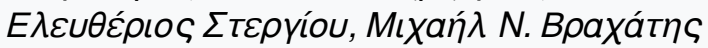

doi: $10.12681 /$ icodl. 662 


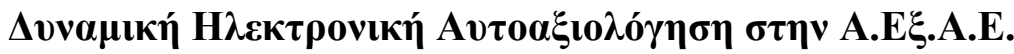

\section{Dynamic Self - Evaluation in O.D.L.}

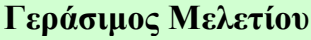 \\ A.T.E.I. H $\pi \varepsilon i ́ p o v$ \\ $\mathrm{K} \alpha \theta \eta \gamma \eta \tau \eta \dot{s}$ \\ gmelet@teiep.gr
}

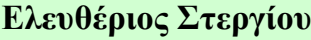

A.T.E.I. H $\pi \varepsilon i ́ p o v$

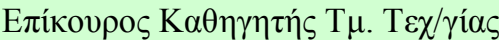

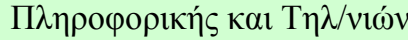

ster@teiep.gr
$\Delta \eta \mu \eta ́ \tau \rho เ o \varsigma ~ B \alpha \sigma ı \lambda \varepsilon เ \alpha ́ \delta \eta \varsigma$

A.T.E.I. H $\pi \varepsilon i ́ \rho 0 v$

K.E. $\Delta$. A.T.E.I. HПEIPOY

dvas@teiep.gr

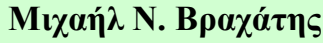

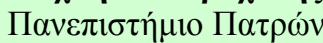

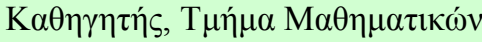

vrahatis@math.upatras.gr

\begin{abstract}
Evaluation is a crucial issue for the improvement of the educational procedure. On the other hand open and distance education mainly relies on web technologies and resources. In the past protocols for secure electronic voting have been used for the functioning of e-evaluation as the security requirements are similar. Techniques that originate from the field of public key cryptography have been employed. In this contribution, e-voting protocols that do not require a Central Tabulating Facility are proposed for the implementation of electronic group self - evaluation in open and distance learning.

\section{Пврі́ $\eta \Psi \psi \eta$}

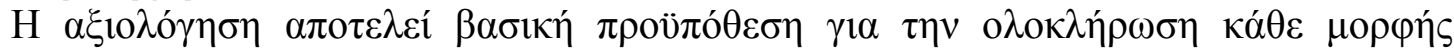

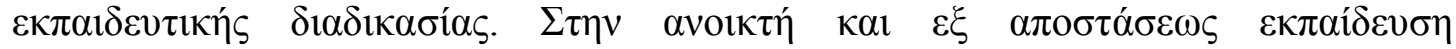

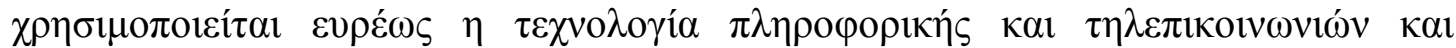

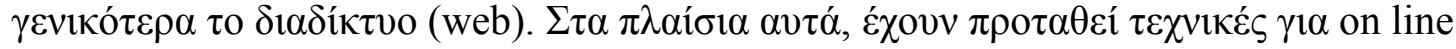

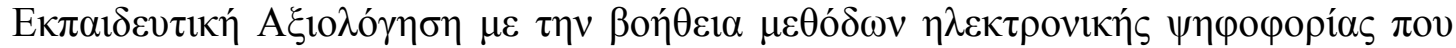

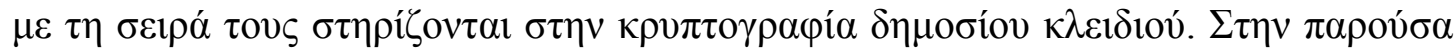

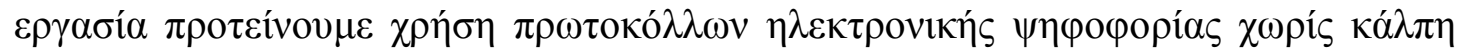

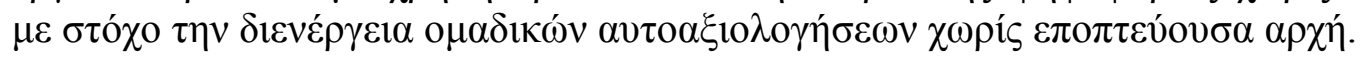

Key-words: open distance learning, poll free e-voting protocol, e-evaluation, public key cryptography, self-evaluation, self-adjudicating protocol.

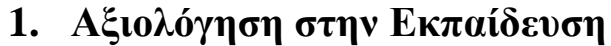

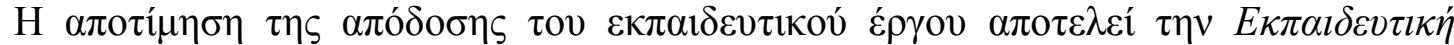

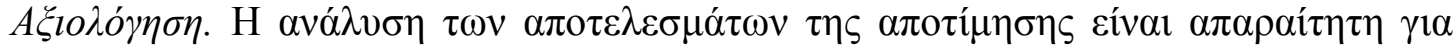

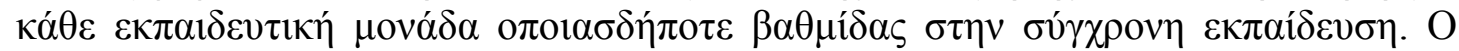

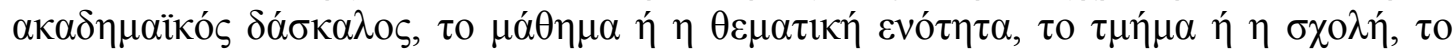

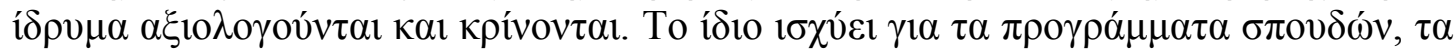

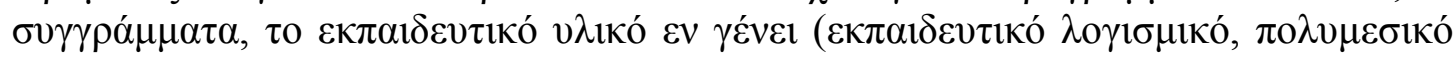

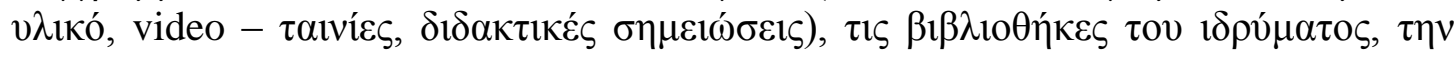




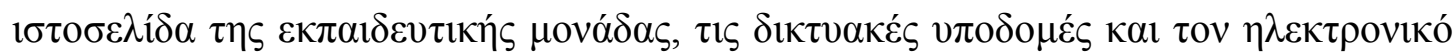

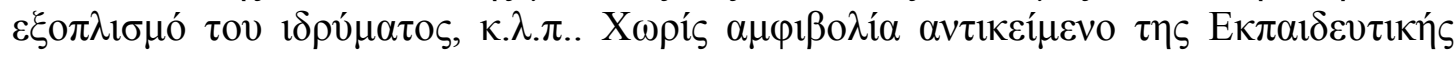

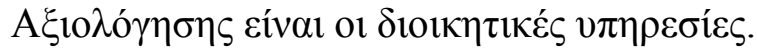

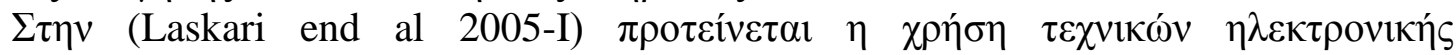

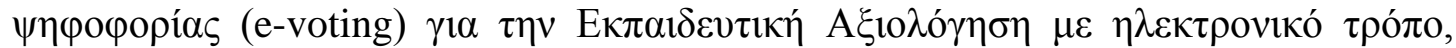

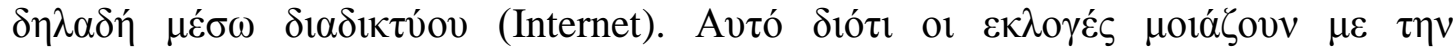

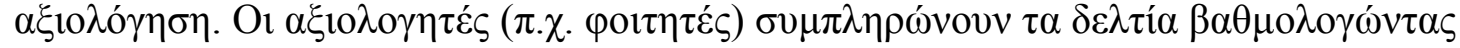

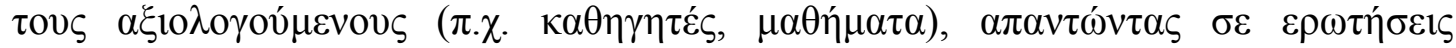

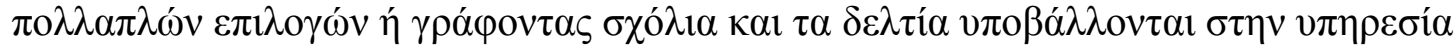

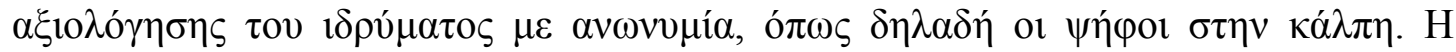

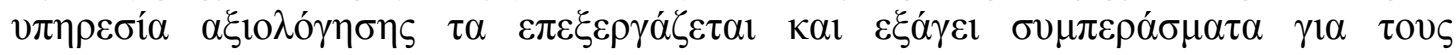

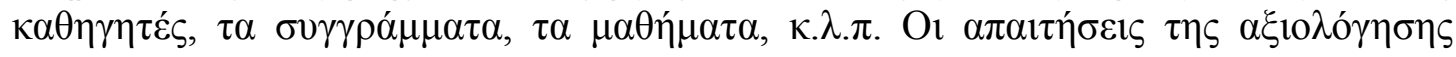
cíval:

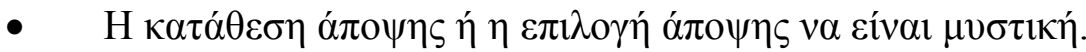

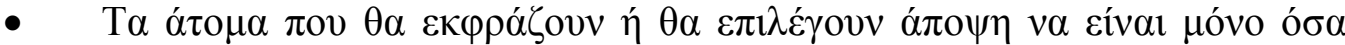

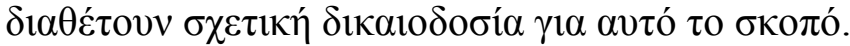

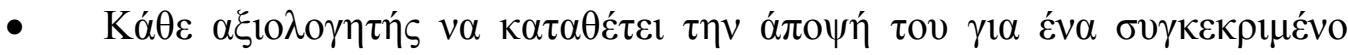
$\theta \dot{\varepsilon} \mu \alpha \alpha \xi$ ı̀

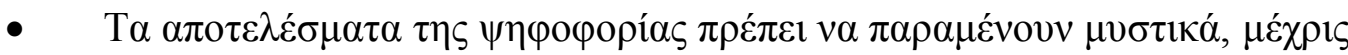

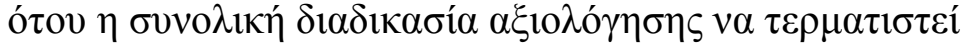

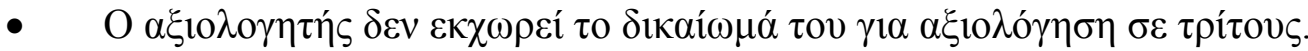

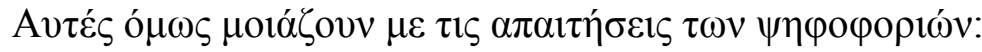

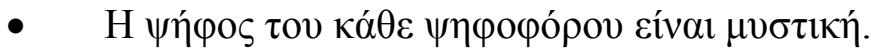

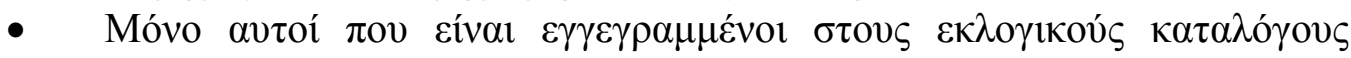

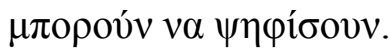

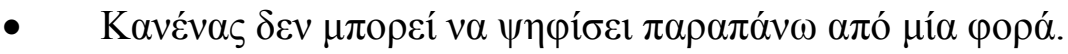

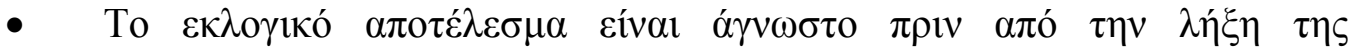

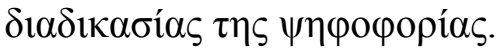

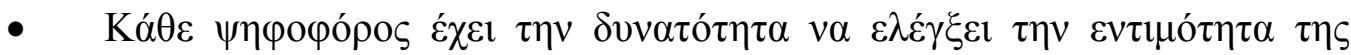

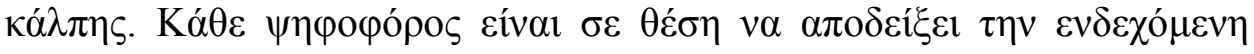

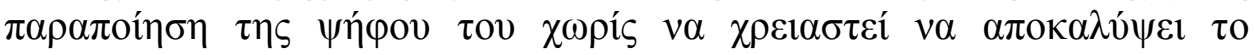

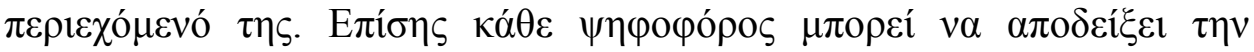

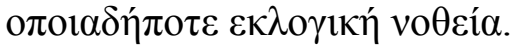

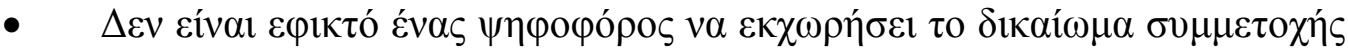

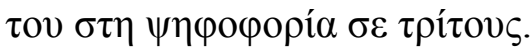

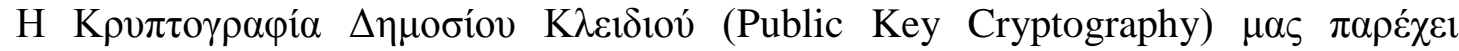

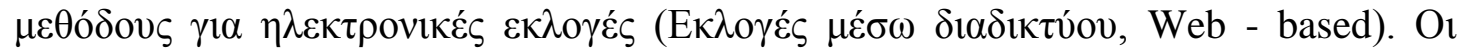

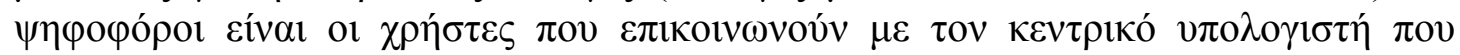

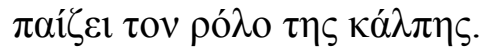

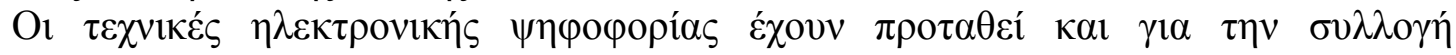

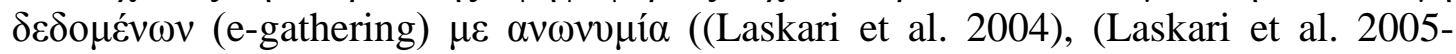

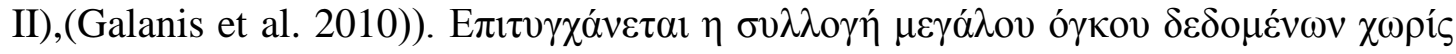

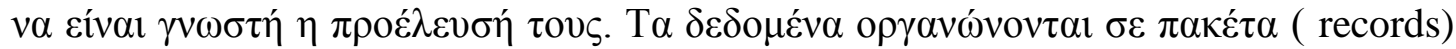

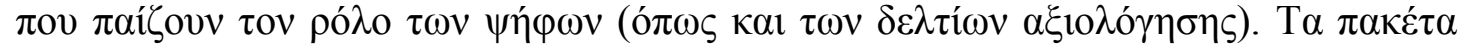

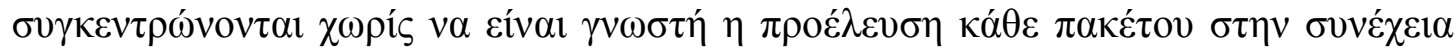

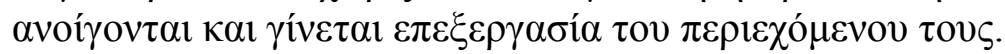




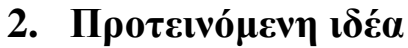

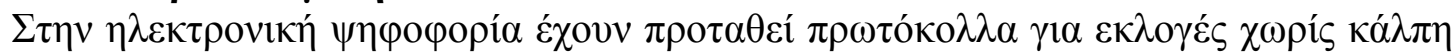

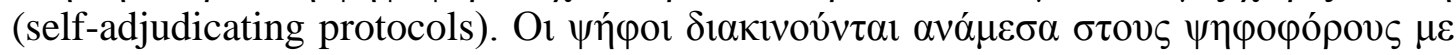

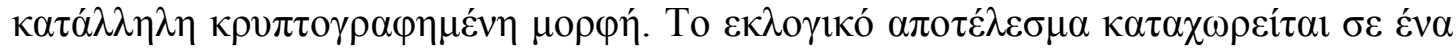
(

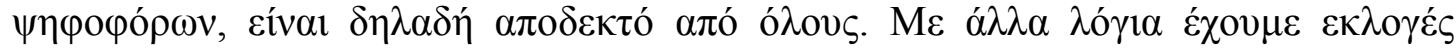

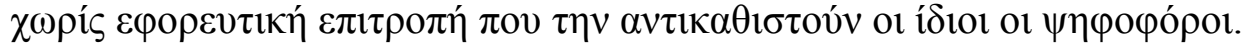

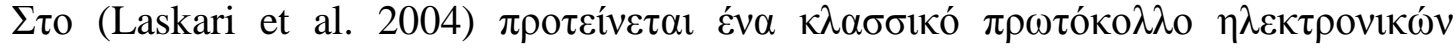

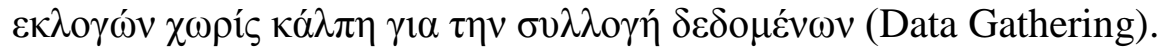

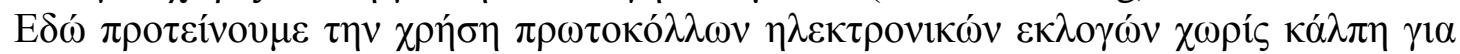

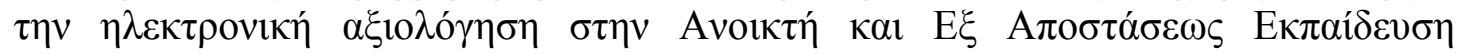

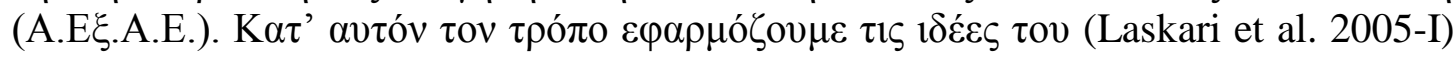

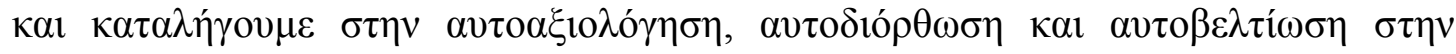
A.E $\xi . A . E .$.

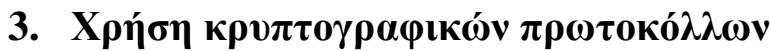

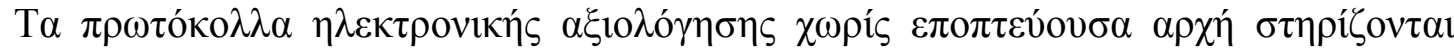

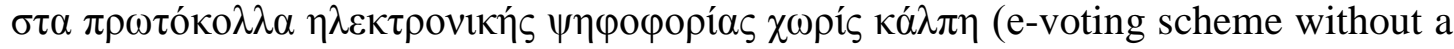

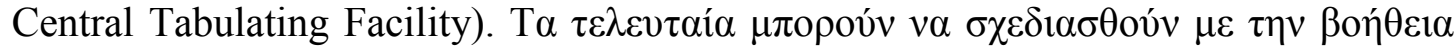

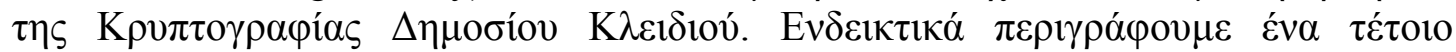

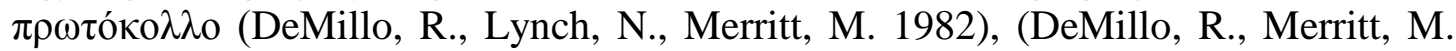
1983), (Chung J. and al. 1998).

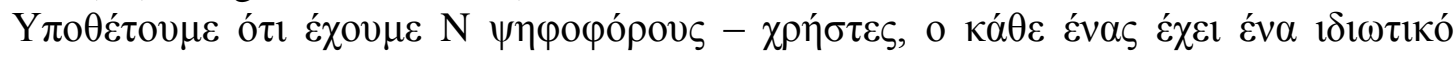

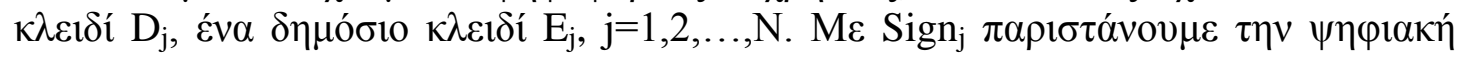

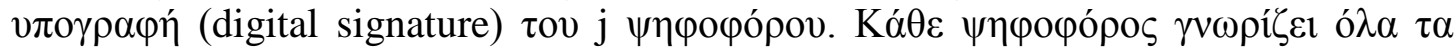

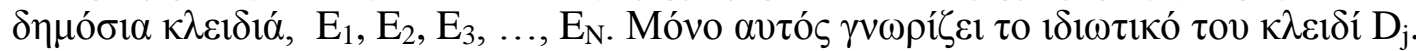

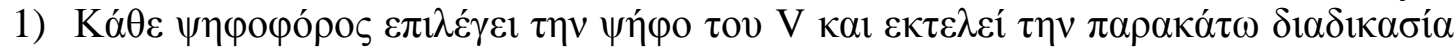
(procedure).

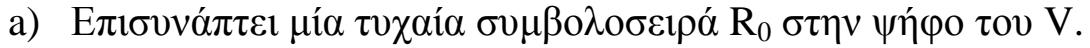

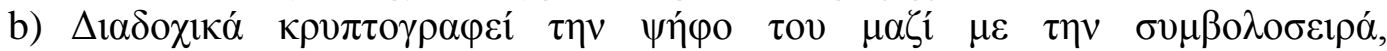

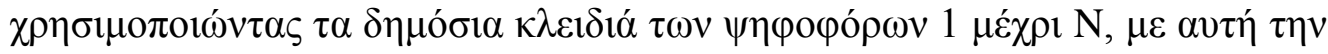

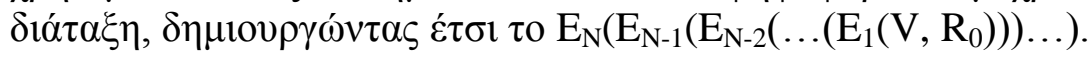

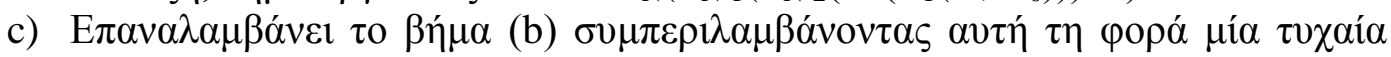

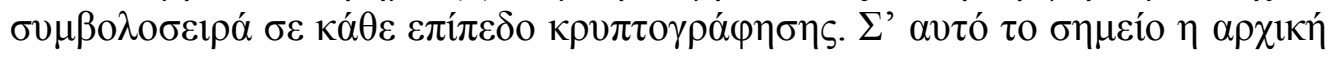

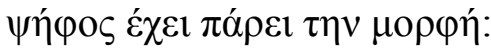

$\mathrm{E}_{\mathrm{N}}\left(\mathrm{R}_{\mathrm{N}}, \mathrm{E}_{\mathrm{N}-1}\left(\mathrm{R}_{\mathrm{N}-1}, \ldots\left(\mathrm{R}_{2}, \mathrm{E}_{1}\left(\mathrm{R}_{1}, \mathrm{E}_{\mathrm{N}}\left(\mathrm{E}_{\mathrm{N}-1}\left(\mathrm{E}_{\mathrm{N}-2}\left(\ldots\left(\mathrm{E}_{1}\left(\mathrm{~V}, \mathrm{R}_{0}\right)\right) \ldots\right)\right)\right)\right) \ldots\right)\right)\right.$ ó $\pi \mathrm{ov}$

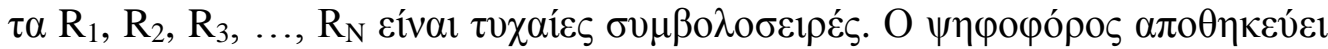

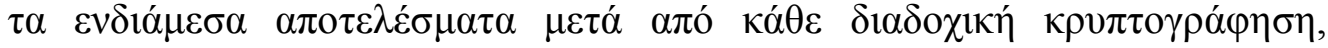

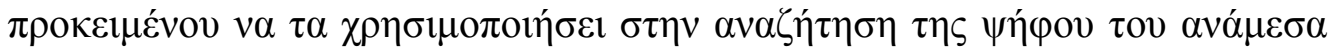

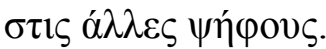

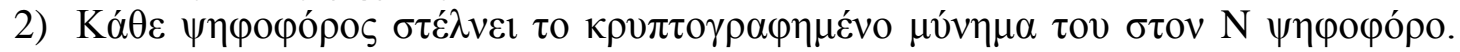

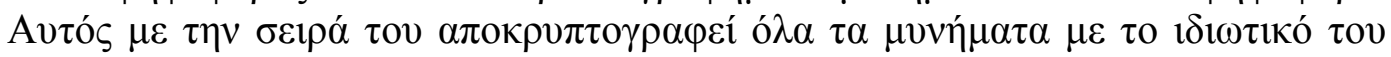

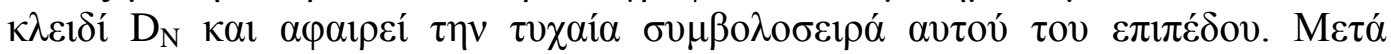

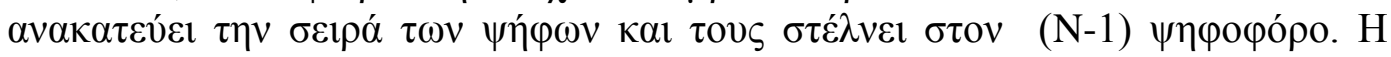

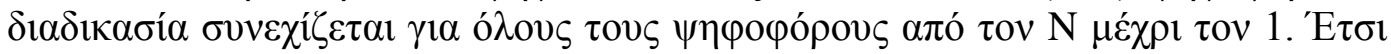

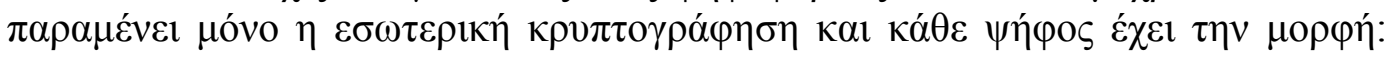
$\mathrm{E}_{\mathrm{N}}\left(\mathrm{E}_{\mathrm{N}-1}\left(\mathrm{E}_{\mathrm{N}-2}\left(\ldots\left(\mathrm{E}_{1}\left(\mathrm{~V}, \mathrm{R}_{0}\right)\right)\right) \ldots\right)\right.$

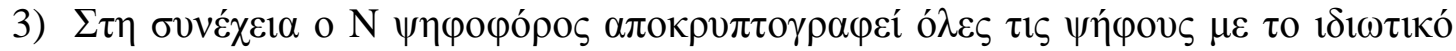

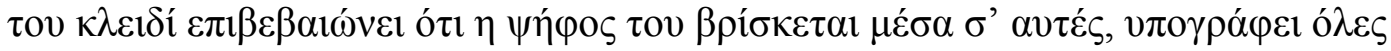

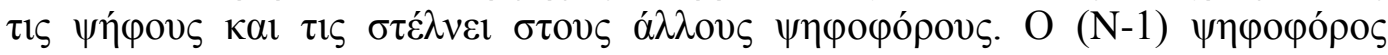




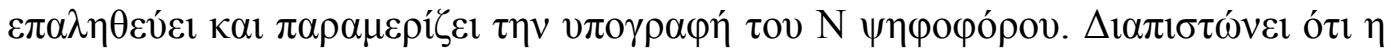

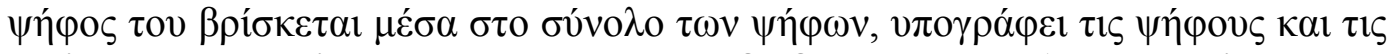

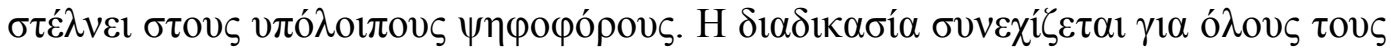

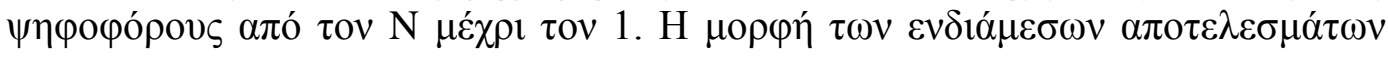

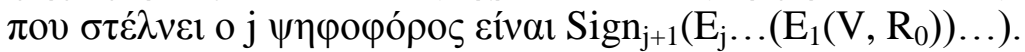

4) 'O

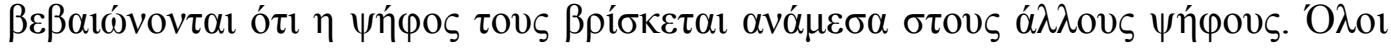

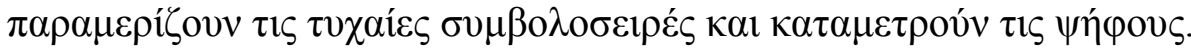

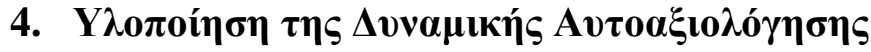

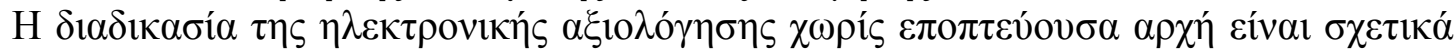
$\alpha \pi \lambda \eta \dot{:}$

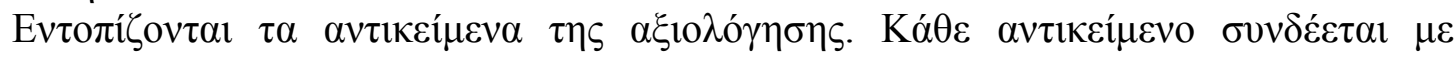

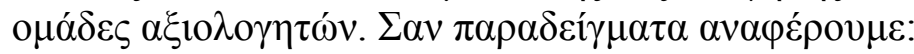

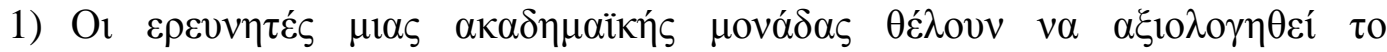

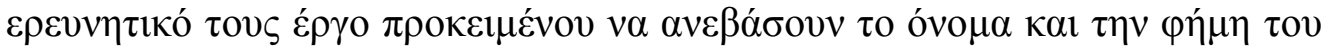

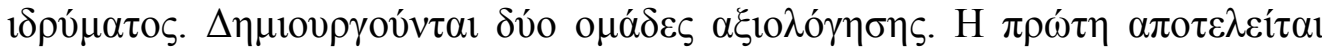

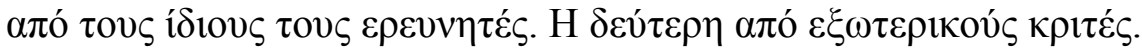

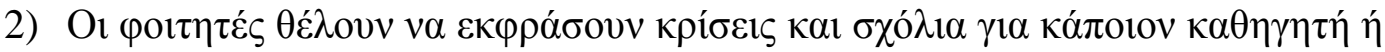

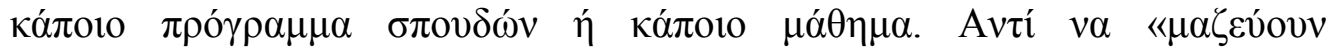

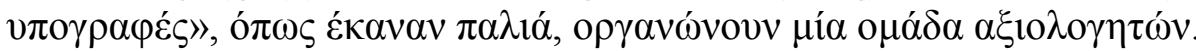

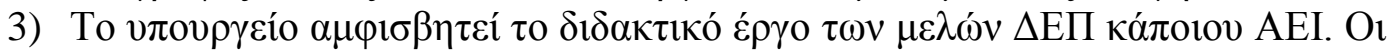

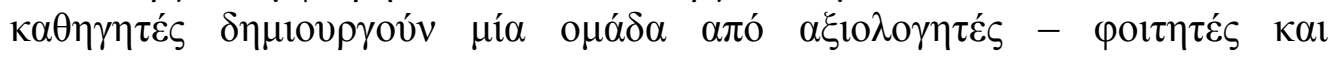
$\pi \rho \circ \alpha \alpha \lambda$ ov́v $\mu$ ía $\alpha \xi 10 \lambda o ́ \gamma \eta \sigma \eta$.

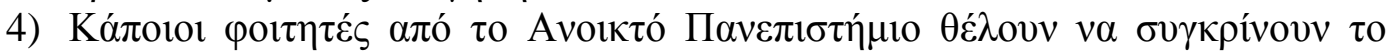

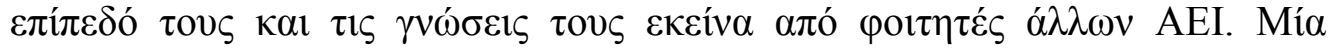

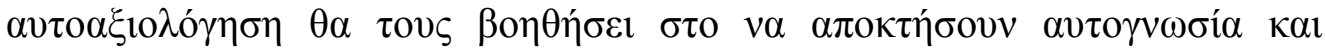
$\varepsilon v \delta \varepsilon \chi 0 \mu \varepsilon \dot{v} \omega \varsigma \alpha v \tau о \pi \varepsilon \pi \circ \dot{\theta} \theta \eta \sigma \eta$.

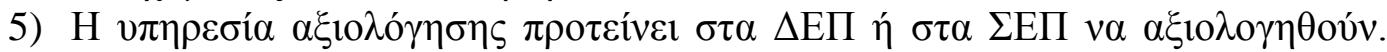

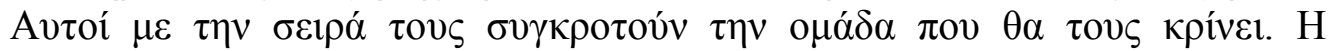

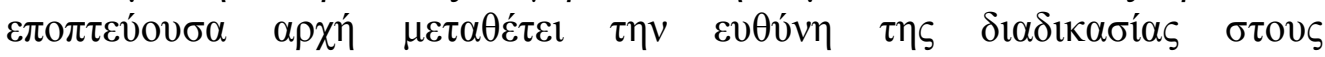

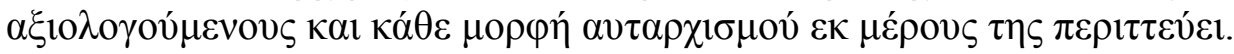

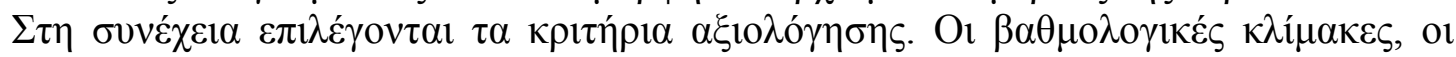

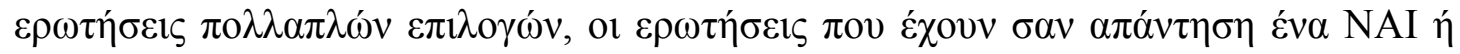

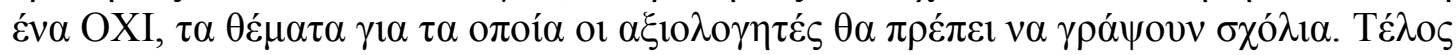

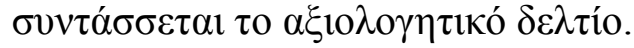

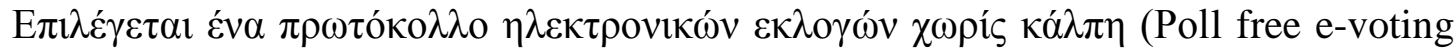

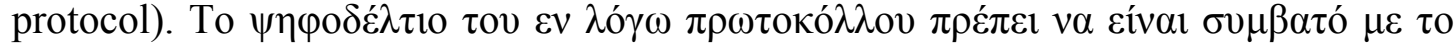
$\alpha \xi$ ıı

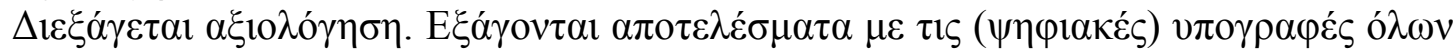
$\tau \omega \nu \alpha \xi 10 \lambda \mathrm{o} \gamma \eta \tau \omega$.

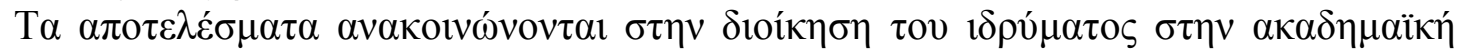

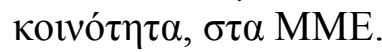

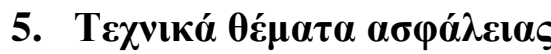

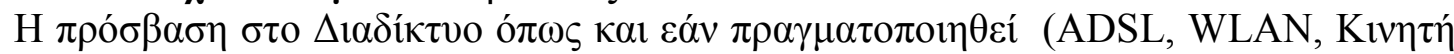

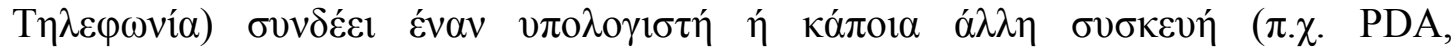

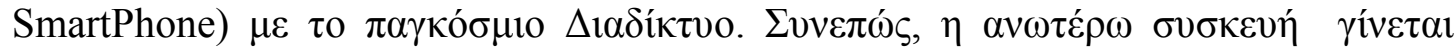

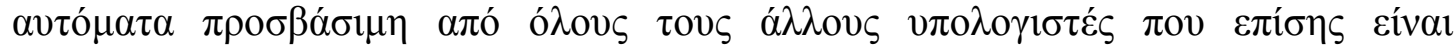

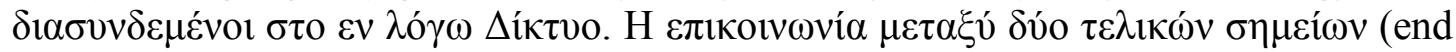




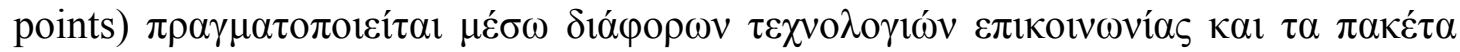

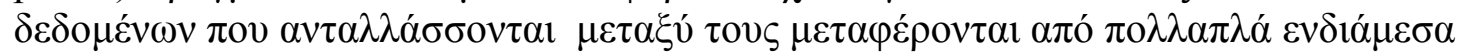

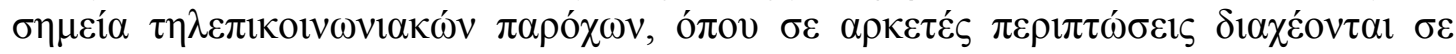

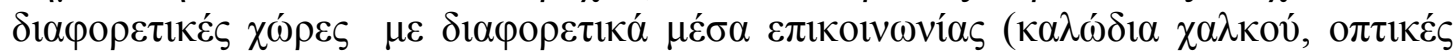

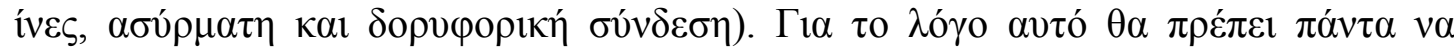

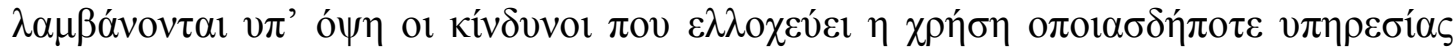

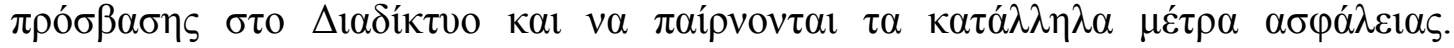

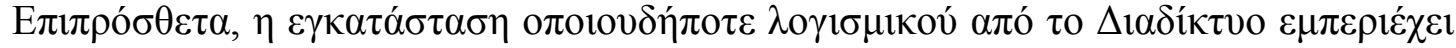

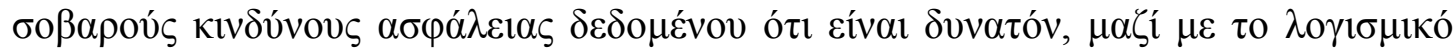

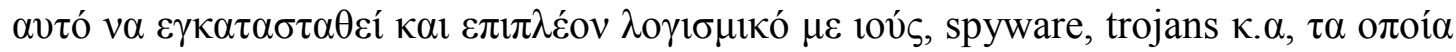

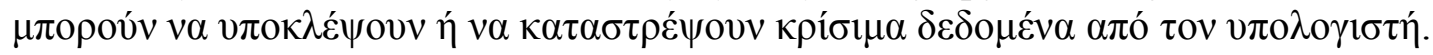

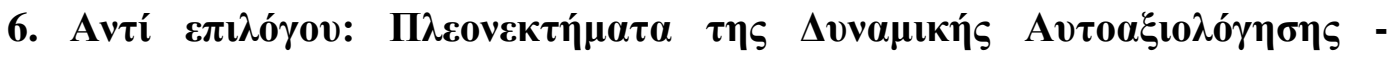 $\Sigma v \mu \pi \varepsilon \rho \alpha ́ \sigma \mu \alpha \tau \alpha$}

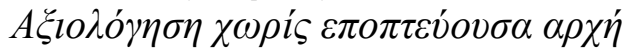

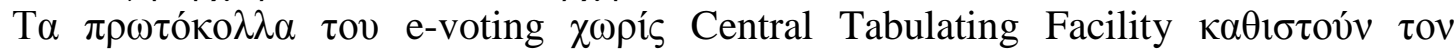

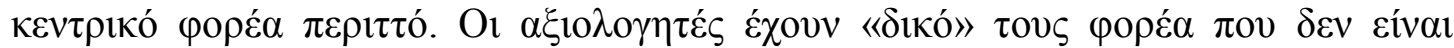

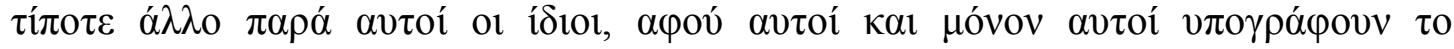

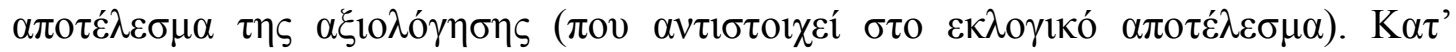

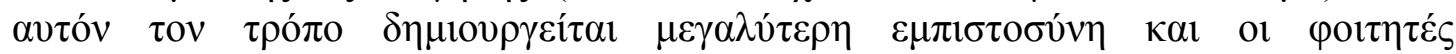

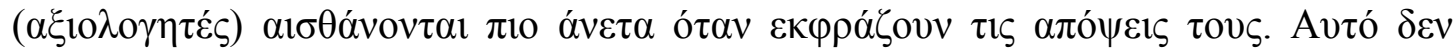

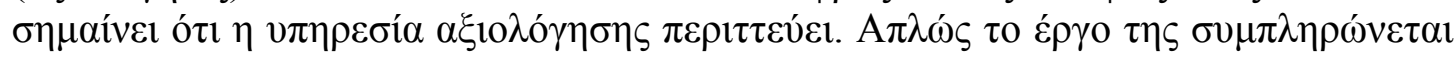
$\alpha \pi$ $\tau 1 \zeta \alpha 0 \tau 0 \alpha \xi ı \lambda o \gamma \eta ́ \sigma \varepsilon ı$.

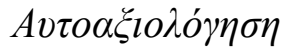

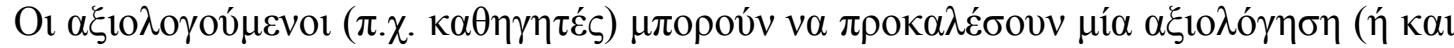

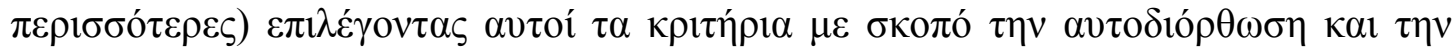

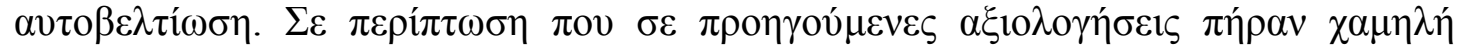

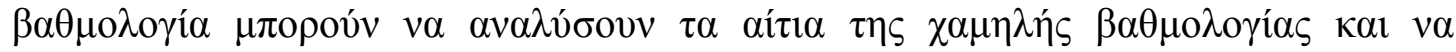

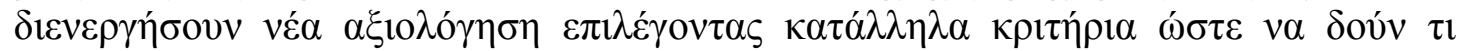

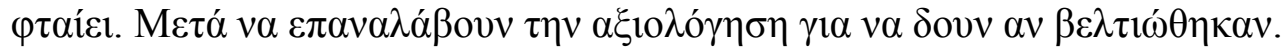

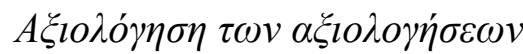

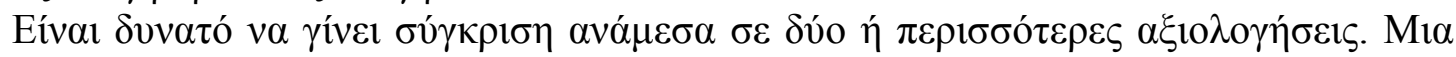

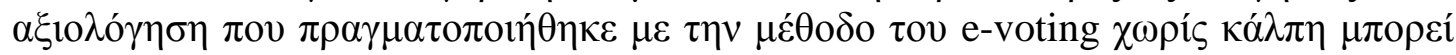

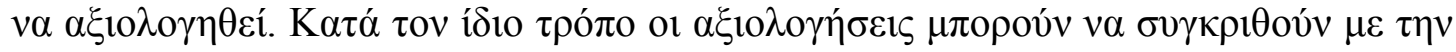

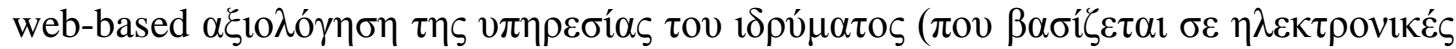

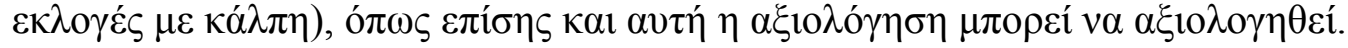

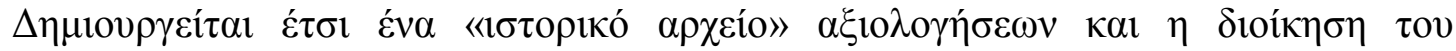

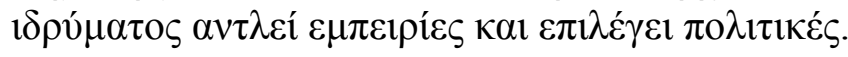

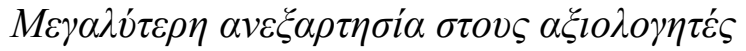

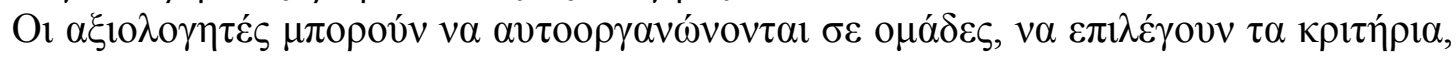

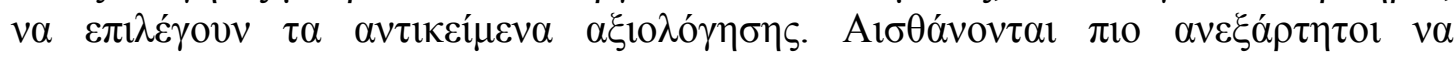

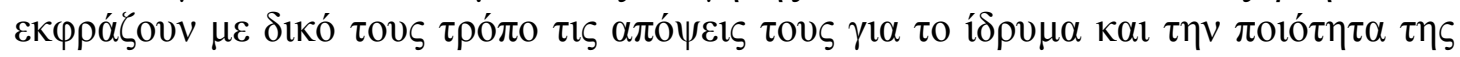
$\varepsilon \kappa \pi \alpha i ́ \delta \varepsilon v \sigma \eta \varsigma \pi 0 v \pi \alpha \rho \varepsilon ́ \chi \varepsilon 1$.

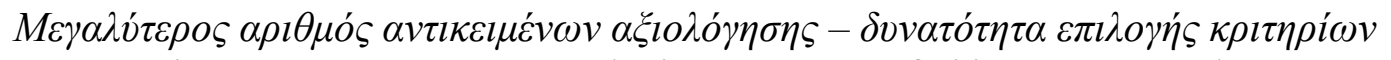

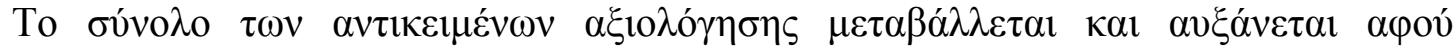

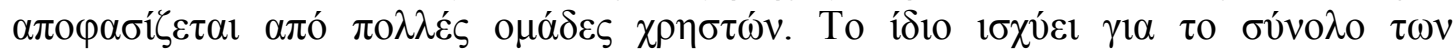




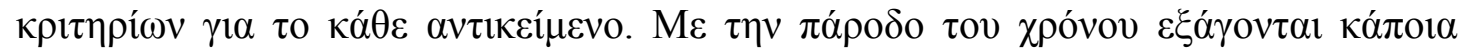

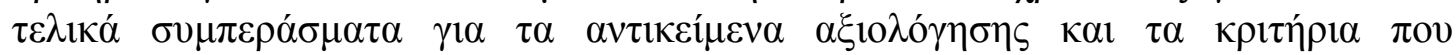
$\alpha \nu \tau \imath \sigma \tau o \iota \chi 0 v ́ v \sigma \varepsilon \kappa \alpha ́ \theta \varepsilon \alpha \nu \tau \imath \kappa \varepsilon i ́ \mu \varepsilon v o$.

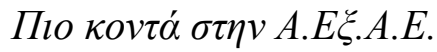

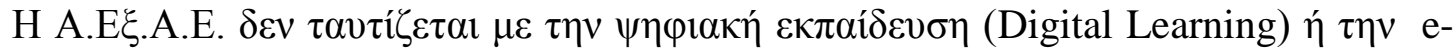

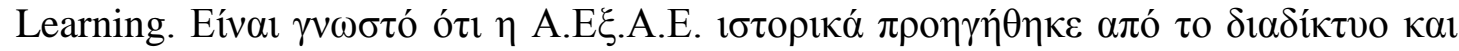

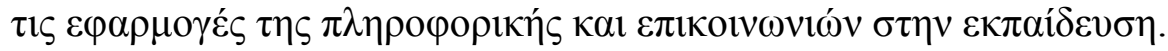

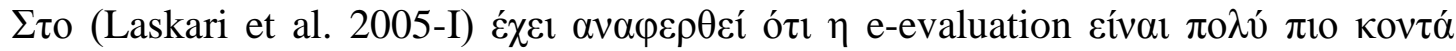

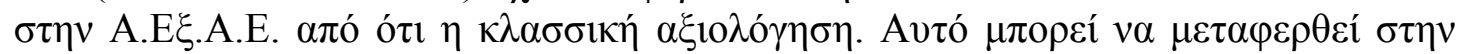

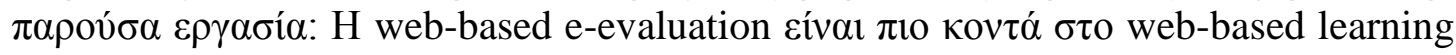

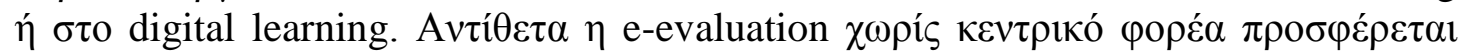

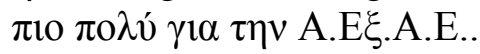

\section{Avapopés}

Chung J. and al. (1998). Democratic Structures in Cyberspace, M.I.T. http://groups.csail.mit.edu/mac/classes/6.805/admin/admin-1998/conf-details/topic10democracy.html

DeMillo, R., Lynch, N., Merritt, M., (1982). Cryptographic protocols, Proceedings of the 4th ACM Symposium on the Theory of Computing, pp. 383-400.

DeMillo, R., Merritt, M., (1983). Protocols for data security, IEEE Computer, Vol. 16, pp. 39-50

Galanis V.I., Ikonomakis E.K., Meletiou G.C. and Vrahatis M.N., (2010). An e-voting based data gathering scheme for decision support systems, International Journal of Decision Sciences, Risk and Management (IJDSRM), Vol. 2, Nos. 1/2, pp. 36-45, Inderscience Enterprises

Laskari E.C., G. C Meletiou and M.N. Vrahatis, (2004), Recent Approaches to Electronic Data Gathering with Privacy, Proceedings of 1st International Conference: "From Scientific Computing to Computational Engineering”, (1 st IC-SCCE, 2004)

Laskari E.C., G. C Meletiou, E. Stergiou and M.N. Vrahatis, (2005). Electronic Evaluation in Open and Distance Education, Proceedings of 3rd International Conference on Open and Distance Learning, (ICODL 2005)

Laskari E.C., G. C Meletiou and M.N. Vrahatis (2005), Privacy Preserving Electronic Data Gathering, Mathematical and Computer Modelling, 42, pp. 739-746, Elsevier 